\title{
Evaluating Biotic Elicitation with Phenylalanine and/or Yeast for Rosemary (Rosmarinus officinals L.) Sustainable Improvement under Traditional and Organic Agriculture
}

\author{
Tarek Elsayed S. A., El Sayed S. A. \\ Radioisotopes Department, Nuclear, Research Center, Egyptian Atomic Energy Authority, Cairo, Egypt \\ Email: dr.tarekelsayed64@gmail.com
}

How to cite this paper: Tarek Elsayed, S.A. and El Sayed, S.A. (2021) Evaluating Biotic Elicitation with Phenylalanine and/or Yeast for Rosemary (Rosmarinus officinals L.) Sustainable Improvement under Traditional and Organic Agriculture. Agricultural Sciences, 12, 273-292. https://doi.org/10.4236/as.2021.123018

Received: February 10, 2021

Accepted: March 27, 2021

Published: March 30, 2021

Copyright $\odot 2021$ by author(s) and Scientific Research Publishing Inc. This work is licensed under the Creative Commons Attribution International License (CC BY 4.0).

http://creativecommons.org/licenses/by/4.0/ (c) (i) Open Access

\begin{abstract}
Rosemary (Rosmrinus officinals) is one of the most important medicinal plants was cultivated for two subsequent seasons field experiment trial (March 2018, 2019) designed as factorial split-plot design with three replicates. The main factor 4 biotic elicitors: control $\left(\mathrm{E}_{1}\right)$, phenylalanine $\left(\mathrm{E}_{2}\right)$, yeast $\left(\mathrm{E}_{3}\right)$ and (E4), E2 + F3 Whereas, the sub-main factor, four fertilizers: (F1) NPK, (F2) $1 / 2 \mathrm{NPK}+\mathrm{PGPB}$ and humic acid + PGPB $\left(\mathrm{F}_{3}\right)$, and moringa dry leaves extract. + PGPB $\left(\mathrm{F}_{4}\right)$. Statistical analysis for collected data revealed significant promotion for growth traits leading to significant increment biomass yield, secondary metabolites production and quality. Total phenolics, total flavonoids and essential oil its terpenes contents in which solitary, E2, E3 acted positive significant impact while E4exhiboted significant positive impact over E1 whereas, F1-4 achieved significant increment in which, biofertilser $\mathrm{f} 4>\mathrm{f} 3>\mathrm{f} 2$ over NPK biofertilser, while paired E1-4 with F1-4 performed E4E4 exceeded E3F3 exceeded E3F2 that exceeded E1F1. Therefore, multi-repeating elicitation with E2, 3, 4 coupled with Biofertilizers F2, 4 could be considered as eco-friendly innovative reliable practical application for sustainable improvement and sustainable use that exceeded significantly over traditional agriculture NPK alone or 1/2 NPK-PGPB for $R$. officinals.
\end{abstract}

\section{Keywords}

Rosemary, Aromatic Plant, Medicinal Plant, Elicitation, Biotic Elicitor

\section{Introduction}

Rosemary (Rosmarin officinalis $\mathrm{L}$ ), RM, is a perenial aromatic and medicinal 
plant belong to the family Lamiaceae and originated from the Mediterranean region. However, it could found all over the world. It may be used as a spice in cooking, as a natural preservative in the food industry, and as ornamental and medicinal plant [1] [2]. RM, is one of the most important aromatic and medicinal plant in the world. It is grown under a wide range of climates, endogenous to Europe. Asia and Afica, mainaly in areas surrounding the Mediterranean Sea [3].

In folk medicine, $R$. officinalis has been used to treat headaches, poor circulation, epilepsy, [4]. Rosemary essential oil (RMEO) was reported to possess strong antioxidant and antimicrobial possess strong antioxidant and antimicrobial properties as wells as wound healing activity [5] [6]. Moreover, topical application of tea tree and RMEO has been documented with satisfactory safety and efficacy [4]-[9]. There is a lot of literature on the usefulness of bioactive supstances of RM plants, in order to the medicinal, pharmaceutical and food industries [10] Therefore many studies were interested in secondary metabolites (SMs) RM plant for their great beneficial effects for human health [11]. RM, has been used as health care supplements to treat arthritis, diabetes, memory loss and hair restoratio [12]. RM has great potential due to the different biological activities of its secondary metabolites (SMs), especially EO and polyphenols which have antidiabctic spasmolytic, carminative, hepatoprotective, antiviral and carcinogenic activities [11]. Furthermore, the aromatic RM herb is added to different types of food to improve the flavor and its organoleptic properties, stringent and food preservative and its antioxidant properties are still uses to extend the shelf-life of prepared foods [13] [14].

$R$. officinalis, can promote several pharmacological effects demonstrated by this plant [2], ability to attenuate asthma, atherosclerosis, cataract, renal, colic, hepatoxcity, peptic ulcer, inflammatory diseases, ischemic heart diseases [15] [16] control of hypercholesterolemia and oxidative stress and relief of physical and mental flatig [17], myocardial blood pressure reduction with rosmarinic acid [16], antiulcer action [18]. Lipid peroxidase reduction in heart and brain, [19]. antiangiocarnosal [20], prevention of problems related to the atherclerosis [21] anti-cancer and antiproliferative effects [22] [23] [24], antiviral [25] and antimicrobial actions [26] hepatoprotective [27], neuroprotective [28] and radioprotiective anti-mutagenic capacities [29], glycaemia reduction [30] muscle relaxant and treatment for cutaneous allergy [31], ability to treat depressive behavior [32], reducting the reactive oxygen (ROS) in Hela cells without cell toxicity [33].

$R$. officinalis, Contains a number of phytochemicals including rosmaric acids console, carnosic and oleanolic acid, unsolic acid. These compounds and some of their derivative have been demonstrated to have multiple physiological activities such as antioxidant [34] [35] anticancer [36] [37]. Anti-diabetic [38] [39] and could be a therapeutic agent in neurodegenerative disease treatment [40] [41] [42]. 
The antioxidant and antimicrobial activities of RM extract are mainly due to carnosic and carnosol [43] [44]. Carnosic acid is the major phenolic diterpene compound in RM leaves that stabilizes unsaturated fatty acids and thus related their deterioration [44] [46] [47], RMEO contain components with insecticidal properties that can be used as pesticides for stored product pests [48], RM extracts could scavenge free radical effects against boldenone induced damage in heart [49]. Also, RM infusion protect against hypodermic-ischemia [50]. RMEO, had bacteriostatic effect at a low concentration which is important for application in the food industry and did not have a germicidal effect on bacterial cells [51].

Elicitation application, can be used to increase SMs production and quality [52] [53] [54] [55] through enhance the transcription of biosynthetic genes involved in SMs biosynthetic pathway [56] [57] [58] that has potential importance particularly on human health benefits [59] [60] and play a major role in adaptation of plants to the changes environmental overcoming biotic and abiotic stresses, [61] [62]. Modified growth and development, can have benifical effects on morphological, physiological, biochemical characteristics than increased biomass yield production and quality [63] [64], Effortful studies has been undertaken for motivtation. innate ability of elicitation in producing extent of bioactive SMs and/or biomass production [65] Alos elicitation has been applied to stimulate the medicinal plants production through organic and agrochemical management contributing [66], Elicitation have related yet distinct role in regulation of plant and pathogen attack, that cause hug loss in yield production under agrochemical traditional agriculture [67], Biotic and abiotic stress/eliators, induces, the production of oxygen derived radicals such as $\mathrm{H}_{2} \mathrm{O}_{2}$ (hydrogen peroxide), superoxide molecules, hydroxyl $(\mathrm{OH})$ and/or oxygen radicals $(\mathrm{O})$ that are the first line of defense for stressed plant [68] which are often implicated to induce systemic resistance (ISR) by regulating the expression genes involved for production and accumulation of SMs, phytoalexins (PAs) which non specific toxins characterized brood spectrum bio-anti-pesticides and bio-anti-micro biocides making them improving against microbial diseases and pests infestation [64] [69] [70] [71].

In the traditional cultivation methods, the excessive use of pesticides and chemical fertilizers leads to an imbalance of nutrient contents in the soil, an increase in vegetative quality and yield. In the face of increasingly serious inviornmental and food problems, organic agriculture is considered to be an effective solution. Since the beginning of green revolution the agriculture has changed by excessive use of fertilizers pesticides, microbiocides [72] [73] in order to increase productivity this agrochemical has been indiscriminately used not only in grain and horticulture plants [66]. Organic agriculture (OA) has been growth in recent years, reaching a 300\% increased of production unite between 2010-2018 [74], this trend, both in production and consumption. Agrochemicals and pesticides impact the environment, preventing sustainable devel- 
opment [75] [76] [77]. Several studies indicated an association between the increase use pesticides and health-related problems such as incidence of fatal malformation, child hood and Juvenile cancer, impacts on hearing loss and numerous occupational contamination events of renal registered in the health system [78] There is a contradiction when talking about the safe use of pesticides, microbiocides for the application of the product [79].

Over recent decades organic farming practices have more widely respected globally, leading to significant increase in certified organic farm [80] [81]. According to the recent survey by [82], a total of 69.8 million hector were organically managed and 93 countries had organic regulation at the end. Organic agriculture practices include a focus on soil health, ecological process and biodiversity without relying on use synthetic chemicals impute [83]. Grown organically medicinal plant by using different organic fertilizers, produced best results in many investigations [84]. It has been reported that organic fertilizers enhanced dry weight, yield, total phenolic, total flavonoids and vitamin C. besides, microorganism can be act as elicitors and increase in the biological and pharmaceutical activities as well as overcoming biotic and abiotic stresses [85]. Such as phenolic flavonoids and terpenes content, microbial activity, chlorophyll content, nutrient uptake, plant growth and development [86] [87] [88] [89]. Biofertilizers has physiological role towards sustainable agriculture in reducing physiological role towards sustainable agriculture in reducing problems associated with the use of agrochemicals [90].

Plant growth promoting bacterium (PGPB) can influence directly or indirectly the general morphology of plants, as they have the ability to recognize tissues and different mechanisms of action [91] [92]. They can act on iron sequestration and phosphorus solubilization, atmospheric nitrogen, hormone production [93], Systemic resistance to pathogens, tolerance to biotic and abiotic stress [94] [95]. Bio fertilizers, suppressed plant diseases, inducing systemic resistance (ISR) against pathogens [71] [95], Diminish environmental concerns associated with the use of synthetic fertilizers to be environmentally co-friendly, the application of bio fertilizers [96] [97], Cabable of improving plant growth, yield production and quality [98], Reducing problems associated with use of chemical fertilizers [90] [99], The application of bio stimulant such as microorganisms rhizobacterium, humic acid, moringa, neem leaves in vivo cultivation of medicinal plants open the opertunity for the development of organic fertilizers for agroecolgoical system, aiming at good quality raw material without pesticides, and/or microbiocides, with, increased concentration of SMs biologically and pharmacologically [100]-[106].

To the best of our knowledge, no findings have been reported on malti-repeating elicitation technology with biotic elicitors for promoting sustainable agriculture medicinal and oromatic plants, under tradiational and organic system. Therefore, the aim of this study was to evaluate potential synergistic elicitation impacts with phenylalanine and yeast to sustainable improvement rosmarene ( $R o-$ sonarins officnals L.) biomas production and quality under traditional and or- 
ganic agriculture systems.

\section{Material and Methods}

\subsection{Biotic-Elicitor Application}

$R$. officinals 2-month old plants were foliarily, with phenylalanine (essential amino acid), $125 \mathrm{ppm}\left(\mathrm{E}_{2}\right)$, yeast, $1.5 \mathrm{~g} / \mathrm{L}\left(\mathrm{F}_{3}\right)$, and (E4) in targeted in tegrated E2 + E3, with Tween 80 , o.1\%, plants were sprayed only with tween 80 solution, as control l (F1).

\subsection{Fertigation Management}

Four fertilizer (F1-4), chemical NPK, $20 \mathrm{~g} / \mathrm{m}^{2}\left(\mathrm{~F}_{1}\right)$, traditional chemical fertilizer, as control $\left(\mathrm{F}_{1}\right)$ and $10 \mathrm{~g} / \mathrm{m}^{2} \mathrm{NPK}$, tinoclulated seeds (F2) with(PGPB) mixture of nitro.fixing bacteria (Azotobacter SP+ Azospirillum $\mathrm{Sp}$ ) and biophosphorus bacteria (Bacillus $\mathrm{Sp}+$ Pseudomonasp). (F3), humic acid, $20 \mathrm{~g} / \mathrm{m}^{2}+$ inoculated seeds with-(PGPB), (F4) moriga dry leave extract, $20 \mathrm{~g} / \mathrm{m}^{2}$, + inoculated Seeds with PGPB. Such F1-4, $30 \mathrm{~m}^{3} / \mathrm{L}$ from solution of 5\% from each Fe, Zn, Mn, Mg, $\mathrm{Cu}$, were added. Fertigation was under taken monthly from sowing up tell one month before harvesting.

\subsection{Execute Field Experiment}

Inoculated and non inoculated seeds with PGPB were cultivated two subsequent seasons field experiment trials, 2018 and of 2019, were designed as factorial split-plot based on randomized complete block design with 3 replications. Four elicitors $\left(\mathrm{E}_{1-4}\right)$ as main plot and 2-bio fertilizers $\left(\mathrm{F}_{3}, \mathrm{~F}_{4}\right)$. RM seeds were sown 20 March at both seasons, in plots $3 \times 2.5 \mathrm{~m}^{2}$ size in rows $50,60 \mathrm{~cm}$ enter and entra-spacing. Irrigation and fertigation management through surface drip irrigation system. Resultant plants aged 60, 90, 120, 150, 180 days were foliarly sprayed with $\left(\mathrm{E}_{1-4}\right)$ and harvested at September 2018, 2019.

\subsection{Biometeric growth traits}

1) Five randomized selected, plants were recorded for plant height $(\mathrm{PH}, \mathrm{Cm})$, number of branches/plant (NBP), fresh herb/plant (FHP, g.) dry herb/plant (DHP, g.), fresh leaves per plant (FLP, g.) and dry leaves per plant (DLP, g.).

2) Biomass yield traits: fresh herb yield, $\mathrm{Kg} / \mathrm{m}^{2}\left(\mathrm{FHY}, \mathrm{Kg} / \mathrm{m}^{2}\right)$, dry herb yield, $\mathrm{Kg} / \mathrm{m}^{2}$ (DHY, $\mathrm{Kg} / \mathrm{m}^{2}$ ), fresh leaves yield, $\mathrm{Kg} / \mathrm{m}^{2}$ (FlY, $\mathrm{Kg} / \mathrm{m}^{2}$ ) and dry leaves yield, $\mathrm{kg} / \mathrm{m}^{2}$ (DLY, $\mathrm{Kg} / \mathrm{m}^{2}$ ) were also recorded.

\subsection{Quli-Quantitative Bioactive Secondary Metabolites (BSMs) Evaluation}

Phenolic Compounds

Extraction procedure:

Dry leaves samples of RM powder (15 g.) were placed in the filter cartridge 
(paper No. 89) in a classical soxhlet apparatus and extracted with $150 \mathrm{ml}$ of an apparatus and extracted with $150 \mathrm{ml}$ of an appropriate solvent for $3 \mathrm{~h}$. for this extraction, two solvents were used, ethanol (100\%) and ethyl acetate (100\%). The samples of RM extracts were stored in glass vials with Teflon sealed, at $20 \pm 0.5$ $\mathrm{C}$ in the absence of light.

Total penolic content (TPC) assay:

TPC was assayed by folin-ciocaleau clorimetric method [107], methanolic extracts $(0.1 \mathrm{ml})$ was mixed with $2.5 \mathrm{ml}$. distilled water followed by the addition of $1 \mathrm{ml}(2 \mathrm{~N})$ folin-Cicalteau ragnet. Then $0.5 \mathrm{ml} 20 \% \mathrm{Na}_{2} \mathrm{CO}_{3}$ was added after 5 min and mixed well the color was developed after $3 \mathrm{~min}$ in the dark at $24^{\circ} \mathrm{C}$ and the absorbance was measured at $760 \mathrm{~nm}$ by vesible spectrophotometer. The absorbance was calibrated using a standard curve with gallic acid and were expressed as mg of gallic acid equivalent per gram dry weight of leaves.

Total flavonoid content (TFC) assay:

TFC was determined calorimetrically using the method described by [90] the methanol leaves extract standard $(0.25 \mathrm{ml})$ were mixed with $1-475 \mathrm{ml}$ distilled water. Ten $0.075 \mathrm{ml} 5 \% \mathrm{NaNO}_{3}$ solutions were added. After $5 \mathrm{~min}$, the absorption was measured at $510 \mathrm{~nm}$ using spectrophotometer the absorbance was expressed as mg. of catechin equivalents per gram dry leaves weight.

\section{Main phenolic compound:}

The rosmarynic acid and carnosic acid content of rosmary dry leaves extract were determined by HPLC conditions of device mobile phase. (A, methanol $+\mathrm{B}$, $10 \mathrm{mM} 850 \mathrm{ml}$. acetic acid) and (150 ml acetonitrile mixture) elution condition linear gradiant, flow $1-1 \mathrm{~mm}^{-1}$, coloumin type Zorbax, $5 \mathrm{Um}$. $15 \mathrm{~cm}$. X4 - 6 $\mathrm{mm}$, detector: waters 2487 daul absorbance UV $285 \mathrm{~nm}$, injection volume $25 \mathrm{UL}$.

\section{Essential oil \% (EO\%):}

EO was determined according to [108] by continuous extraction (Soxilet) with acetone. The volatile oil solution obtained is evaporated under reduced pressure, in rotatory evaporator. The oil was weighted and stored in amber colored bottles at $20^{\circ} \mathrm{C}$ til to the farther analysis.

Essential oil yield, g. $/ \mathrm{m}^{2}$ (EOY g. $\left./ \mathrm{m}^{2}\right)$ :

EOY, $\mathrm{g} / \mathrm{m}^{2}$ were determined by multiplying dry leaves yield, $\mathrm{g} . / \mathrm{m}^{2}$ with $\mathrm{EO} \%$.

\section{Essential oil contents:}

Compositions of EO were determined by GC-FID and GC-MS analyses they were achieved on an Agillary Technologies 7890GC equipped with FID and mass spectrophotometer detectors using a HP-5MS $5 \%$ capillary column $(30.00 \mathrm{~m}$ $\mathrm{X} 0.25,0.25 \mu \mathrm{m}$ film thicknesses). The carrier gas was belium at a flow of 0.8 $\mathrm{ml} / \mathrm{min}$. Initial column temperature was $60^{\circ} \mathrm{C} / \mathrm{min}$. the split ratio was $40: 1$. The injector temperature was set at $300^{\circ} \mathrm{C}$. The acquisition range was $50-550 \mathrm{~m} / \mathrm{Z}$ in electron impact (EI) mode using an ionization voltage of 70ev. The assential oils were diluted 1:100 in $\mathrm{n}$-hexan, then $0.1 \mu \mathrm{L}$ were injected into GC systems.

\section{Identification of EO components:}

Identification of the components were performed on the bases of retention 
index (RI), determined with reference of the homologous series of n-alkones, C2-C30, under identical experimental conditions, comparing with the mass spectra library search (NIST and wiley), and wit the mass spectra literature date [109]. The relative amounts of individual components were calculated based on CG peak area (FID response).

\section{Statistical analysis:}

Statistical analysis for the interaction between two subsequent season (2019 and 2021) were found to be not significant. Therefore, The pooled mean values of 2 years for all the traits were subjected to statistical analysis of variance was done for all traits whereas, the calculated least significant differences, LSD at $1 \%$ level were used for comparison between mean treatments.

\section{Results and Discussion}

\subsection{Growth Traits}

Multi-repeating elicitation with $\mathrm{E}_{1-4}$ under $\mathrm{F}_{1-4}$ caused significant positive impact on growth traits $E_{3}>E_{2}>E_{1}$ while $E_{4}$ actuated significant synergistic impact at $\mathrm{F}_{4}>\mathrm{F}_{3}>\mathrm{F}_{2}>\mathrm{F}_{1}$, therefore, the interaction, $\mathrm{F}_{4} \mathrm{E}_{4}>\mathrm{F}_{3} \mathrm{E}_{3}>\mathrm{F}_{2} \mathrm{E}_{2}>\mathrm{F}_{1} \mathrm{E}_{1}$ as represented in Table 1, exceedingly multi-repeating elicitation under bio fertilizer $\left(\mathrm{F}_{3,4}\right)$ over traditional $\left(\mathrm{F}_{2,1}\right)$ Chemical fertilizers, However PGPB, application reduced NPK (F1) these data were in accordance with that has been reported by [4] [97] [110] [111] [112] [113] [114].

\subsection{Biomass Yield Traits}

$\mathrm{E}_{2,3}$ invoked significant increment in DLY, $\mathrm{g} / \mathrm{m}^{2}$ and EOY, $\mathrm{g} / \mathrm{m}^{2}$ whereas $\mathrm{E}_{4}$ achieved synergistic positive impact under $\mathrm{F}_{1-4}$ aside $\mathrm{F}_{1-4}$ resulted in significant improvement aside $\mathrm{F}_{4}>\mathrm{F}_{3}>\mathrm{F}_{2}>\mathrm{F}_{1}$ hence, $\mathrm{F}_{4} \mathrm{E}_{4}>\mathrm{F}_{3} \mathrm{E}_{3}>\mathrm{F}_{2} \mathrm{E}_{2}>\mathrm{F}_{1} \mathrm{E}_{1}$ significantly as represented Table 2 and illustrated Figure 1. These obtained data were in agreement with that has been investigated. [58] [110] [111] [115].

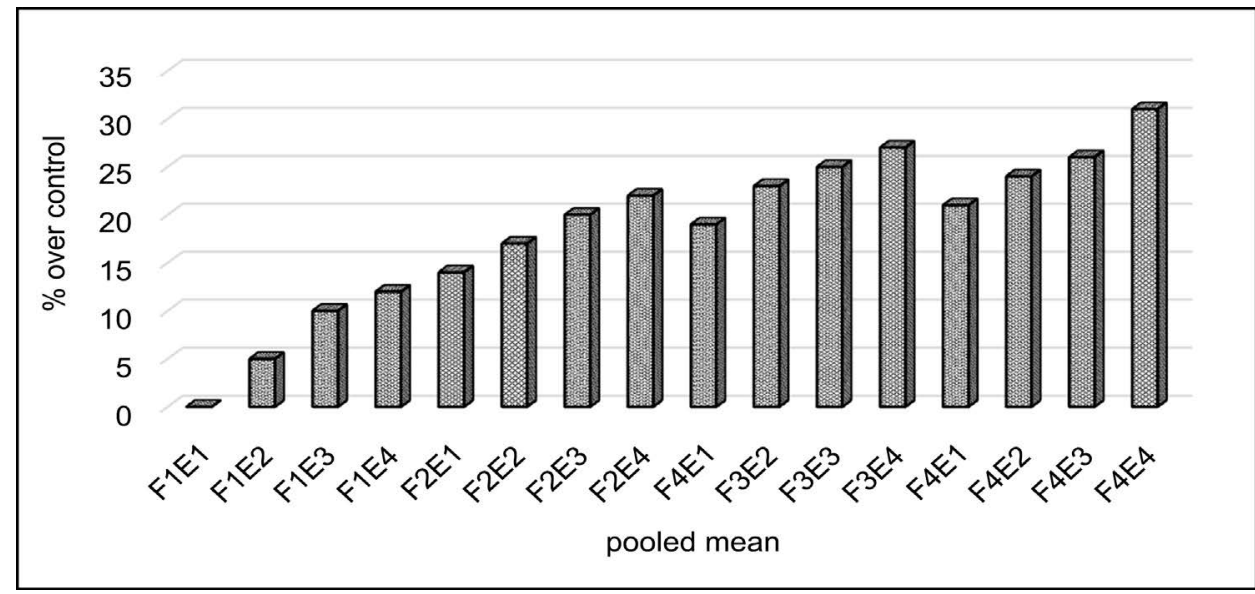

Figure 1. Pooled mean DLY, $\mathrm{g} / \mathrm{m}^{2}$ as \% over control under E1-4 interacted with F1-4 for $R$. officinals. 
S. A. Tarek Elsayed et al.

Table 1. Mean growth traits at two subsequent seasons for $R$. officinals in response to multi-repeating elicitation with 4 biotic elicitors under 2-traditional fertilizer $(\mathrm{F} 1,2)$ and 2-organic, biofertilizers (F3, 4).

\begin{tabular}{cccccccccc}
\hline \multirow{2}{*}{$\begin{array}{c}\text { Application } \\
\text { Treatment }\end{array}$} & \multicolumn{3}{c}{ Plant hight, cm } & \multicolumn{3}{c}{ Number branches } & \multicolumn{3}{c}{ FLYP, g. } \\
\cline { 2 - 9 } $\mathrm{F}_{1} \mathrm{E}_{1}$ & $\mathbf{2 0 1 8}$ & $\mathbf{2 0 1 9}$ & Pooled mean & $\mathbf{2 0 1 8}$ & $\mathbf{2 0 1 9}$ & Pooled mean & $\mathbf{2 0 1 8}$ & 2019 & Pooled mean \\
2 & 70.7 & 98.7 & $84.70(0)$ & 95.8 & 102.5 & $99.15(0)$ & 350.5 & 432.3 & $391.4(0)$ \\
3 & 75.6 & 105.6 & $90.60(+7)$ & 100.6 & 107.6 & $104.10(+5)$ & 368.3 & 435.9 & $411.1(+5)$ \\
4 & 78.5 & 107.9 & $92.70(+9)$ & 104.4 & 111.7 & $108.05(+9)$ & 385.5 & 475.5 & $430.5(+10)$ \\
$\mathrm{F}_{2} \mathrm{E}_{1}$ & 79.8 & 111.5 & $95.65(+13)$ & 111.1 & 118.9 & $115.00(+16)$ & 399.6 & 492.8 & $446.2(+14)$ \\
2 & 81.3 & 113.5 & $97.40(+15)$ & 114.9 & 123.0 & $118.95(+20)$ & 410.1 & 505.8 & $457.9(+17)$ \\
3 & 82.7 & 115.5 & $99.10(+17)$ & 120.7 & 129.2 & $124.95(+26)$ & 420.6 & 518.7 & $469.7(+20)$ \\
4 & 86.8 & 118.4 & $101.60(+20)$ & 125.4 & 134.2 & $129.80(+31)$ & 427.6 & 527.5 & $477.6(+22)$ \\
\hline $\mathrm{F}_{3} \mathrm{E}_{1}$ & 86.3 & 120.4 & $103.35(+22)$ & 116.8 & 125.1 & $120.90(+22)$ & 417.9 & 514.4 & $466.2(+19)$ \\
2 & 88.3 & 123.4 & $105.85(+25)$ & 121.6 & 130.2 & $125.90(+27)$ & 431.1 & 531.7 & $481.4(+23)$ \\
3 & 87.8 & 125.3 & $107.55(+27)$ & 126.4 & 135.3 & $130.85(+32)$ & 438.1 & 540.4 & $489.3(+25)$ \\
4 & 92.6 & 129.3 & $110.95(+31)$ & 129.3 & 138.4 & $133.85(+35)$ & 445.1 & 549.2 & $497.2(+27)$ \\
\hline $\mathrm{F}_{4} \mathrm{E}_{1}$ & 89.2 & 124.3 & $106.75(+26)$ & 122.6 & 131.2 & $126.90(+28)$ & 424.1 & 523.1 & $473.8(+21)$ \\
2 & 91.2 & 127.3 & $109.25(+29)$ & 127.4 & 136.3 & $131.85(+33)$ & 434.6 & 536.1 & $485.4(+24)$ \\
3 & 93.3 & 130.2 & $111.75(+32)$ & 130.2 & 139.4 & $134.80(+36)$ & 441.6 & 544.7 & $493.2(+26)$ \\
4 & 95.4 & 133.2 & $114.30(+35)$ & 132.2 & 141.5 & $136.85(+38)$ & 459.2 & 566.3 & $5128(+31)$ \\
\hline LSD1\% & $\mathbf{0 . 2 3}$ & $\mathbf{0 . 2 8}$ & $\mathbf{0 . 2 6}$ & $\mathbf{0 . 2 5}$ & $\mathbf{0 . 3 1}$ & $\mathbf{0 . 2 7}$ & 1.9 & 2.6 & 2.4 \\
\hline
\end{tabular}

E1-4: O control, phenylalanine yeast and integrated with phenylalanine yeast, respectively; F1-4 NPK chemical, traditional fertilizer, 1/2NPK + PGPB, humic acid + PGPB, and moringa dry leaves extract + PGPB, respectively.

Table 2. Dry leave yield, $\mathrm{g} / \mathrm{m}^{2}\left(\mathrm{DLY}, \mathrm{g} / \mathrm{m}^{2}\right)$ and essential oil yield, $\mathrm{g} / \mathrm{m}^{2}$ (EOY, $\mathrm{g} / \mathrm{m}^{2}$ ) for. $R$. officinalis at 2-subsequent cultivated seasns under traditional and organic fertilizer and multi-repeating illicitation with biotic elicitors.

\begin{tabular}{|c|c|c|c|c|c|c|}
\hline \multirow{2}{*}{$\begin{array}{l}\text { Application } \\
\text { Treatment }\end{array}$} & \multicolumn{3}{|c|}{$\mathrm{DLY}, \mathrm{g} . / \mathrm{m}^{2}$} & \multicolumn{3}{|c|}{ EO DlY, g. $/ \mathrm{m}^{2}$} \\
\hline & 2018 & 2019 & Pooled mean & 2018 & 2019 & Pooled mean \\
\hline $\mathrm{F}_{1} \mathrm{E}_{1}$ & 337.9 & 335.2 & $346.5(0)$ & 273.8 & 301.8 & $387.8(100)$ \\
\hline 2 & 354.8 & 372.9 & $363.8(+5)$ & 445.6 & 522.2 & 483.9 (125) \\
\hline 3 & 371.7 & 390.7 & $381.2(+10)$ & 550.6 & 563.2 & 556.9 (144) \\
\hline 4 & 378.4 & 397.8 & $388.1(+12)$ & 582.1 & 644.8 & $613.4(158)$ \\
\hline $\mathrm{F}_{2} \mathrm{E}_{1}$ & 385.2 & 404.9 & $395.0(+14)$ & 429.3 & 490.1 & 459.7 (119) \\
\hline 2 & 395.3 & 415.5 & $405.4(+17)$ & 549.1 & 607.4 & $578.3(149)$ \\
\hline 3 & 405.5 & 426.2 & $415.8(+20)$ & 641.4 & 707.2 & $674.3(174)$ \\
\hline 4 & 412.2 & 433.3 & $422.7(+22)$ & 688.0 & 740.4 & $714.2(184)$ \\
\hline $\mathrm{F}_{3} \mathrm{E}_{1}$ & 402.1 & 433.3 & $412.4(+19)$ & 570.8 & 630.3 & 600.5 (155) \\
\hline 2 & 415.6 & 436.9 & $426.2(+23)$ & 673.9 & 742.9 & $708.4(183)$ \\
\hline 3 & 422.4 & 440.0 & $433.1(+25)$ & 742.7 & 783.2 & 762.9 (197) \\
\hline 4 & 429.1 & 451.1 & $440.0(+27)$ & 797.9 & 816.3 & 807.1 (208) \\
\hline $\mathrm{F}_{4} \mathrm{E}_{1}$ & 408.8 & 429.7 & $419.3(+21)$ & 621.7 & 683.7 & $652.7(168)$ \\
\hline 2 & 418.9 & 440.4 & $429.6(+24)$ & 691.4 & 743.6 & 717.5 (185) \\
\hline 3 & 425.7 & 447.5 & $436.5(+26)$ & 813.6 & 828.8 & $821.2(212)$ \\
\hline 4 & 442.6 & 465.3 & $453.9(+31)$ & 890.4 & 897.5 & $894.0(231)$ \\
\hline LSD1\% & 11.1 & 12.7 & 9.4 & 9.1 & 11.3 & 10.6 \\
\hline
\end{tabular}

E1-4: O control, phenylalanine yeastand coubled phenylalanine and yeast, respectively; F1-4 NPK chemical, traditional fertilizer, 1/2NPK + PGPB, humic acid + PGPB, and moringa dry leaves extract + PGPB, respectively. 
There is no best and disease incidence in the 2 seasons field experiments, in accordance to multi-repeating elicitation with E2-4 and F2-4, whom could induce systemic resistance (ISR) that lead to protect and biological control for elicitated and bio fertilized plants [69] [100] [102] [111] [113] [116] [117].

\subsection{Biomas Yield Quality Traits}

Secondary metabolites (SMs), total phenol content (TPC), total flavonoid content (TFC). Table 3 and major phenolic acid, romasrynic acid (RCA) and carnosic (CTC). Table 4 declared that E3 $>2$ achieved significant increase for (TPC), TFC, RCA, CTC while E4 resulted synergistic increament in these traits under $\mathrm{F}_{1}-\mathrm{F}_{4}$ aside $\mathrm{F}_{4} \mathrm{E}_{4}>\mathrm{E}_{3} \mathrm{~F}_{3}>\mathrm{E}_{2} \mathrm{~F}_{2}>\mathrm{E}_{1} \mathrm{~F}_{1}$ significantly, as represented (Table 3, Table 4).

SMs, EO components were listed Table 5 that declared, ciniol $(20.33 \%$, linolool (16.57\%), a-penine (15.50), compere (5.80\%), limonine (3.22\%), P-cymene (2.42\%), tepineol (2.82\%), caryophllene (1.70\%) and terpiniol (1.51\%). E2,3 performed significant increase ciniol, liniol, $\alpha$-penine as well as total components for EO while $\mathrm{E} 4$ resulted synergetic increment under $\mathrm{F}_{1-4}$. Aside $\mathrm{E}_{4} \mathrm{~F}_{4}>\mathrm{E}_{3} \mathrm{~F}_{3}>$ $\mathrm{E}_{2} \mathrm{~F}_{2}>\mathrm{E}_{1} \mathrm{~F}_{1}$ significantly. As represented Figure 2 It has been extensively declared that biotic elicitor enhanced SMs production and quality [71] [100] [102]. Also, bio fertilizer enhanced SMs production and quality [110] [111] [118] Since plant pathogens cause huge yield losses. Plant defense often depends in toxic SMs that inhibit pathogen and overcoming biotic and abiotic stresses [69] [70] [110] [119] [120].

Table 3. Total phenolics compound/ and total flavonoids (TFC) compounds for $R$. Offcinalis cultivated 2-subsequent seasns under traditional and organic system in respone to multi repeating biotic elicittion.

\begin{tabular}{|c|c|c|c|c|c|c|}
\hline \multirow{2}{*}{$\begin{array}{l}\text { Application } \\
\text { Treatment }\end{array}$} & \multicolumn{3}{|c|}{ TPC } & \multicolumn{3}{|c|}{ TFC } \\
\hline & 2018 & 2019 & Pooled mean & 2018 & 2019 & Pooled mean \\
\hline $\mathrm{F}_{1} \mathrm{E}_{1}$ & 30.47 & 35.75 & $33.11(100)$ & 2.66 & 3.72 & $3.19(100)$ \\
\hline 2 & 41.13 & 48.26 & 44.69 (135) & 3.45 & 4.83 & $4.14(130)$ \\
\hline 3 & 47.53 & 55.77 & $51.65(156)$ & 3.59 & 5.02 & $4.30(135)$ \\
\hline 4 & 53.32 & 62.56 & $57.94(175)$ & 3.77 & 5.28 & $4.52(142)$ \\
\hline $\mathrm{F}_{2} \mathrm{E}_{1}$ & 31.99 & 37.53 & $34.76(105)$ & 3.05 & 4.27 & $3.66(115)$ \\
\hline 2 & 45.40 & 53.26 & 49.33 (149) & 3.64 & 5.09 & 4.37 (137) \\
\hline 3 & 51.18 & 60.06 & $55.62(168)$ & 3.85 & 5.39 & $4.62(145)$ \\
\hline 4 & 55.45 & 65.06 & $60.25(182)$ & 4.01 & 5.61 & $4.81(151)$ \\
\hline $\mathrm{F}_{3} \mathrm{E}_{1}$ & 33.82 & 39.68 & $36.75(111)$ & 3.21 & 4.50 & $3.85(121)$ \\
\hline 2 & 49.36 & 57.91 & $53.63(162)$ & 3.83 & 5.35 & $4.59(144)$ \\
\hline 3 & 54.23 & 63.63 & $58.93(178)$ & 3.99 & 5.58 & $4.78(150)$ \\
\hline 4 & 58.80 & 68.99 & $63.90(193)$ & 4.44 & 6.21 & $532(167)$ \\
\hline $\mathrm{F}_{4} \mathrm{E}_{1}$ & 35.95 & 42.18 & $39.06(118)$ & 3.32 & 4.65 & $3.98(125)$ \\
\hline 2 & 52.71 & 61.84 & $57.28(173)$ & 4.20 & 5.87 & $5.04(158)$ \\
\hline 3 & 56.36 & 66.13 & $61.25(185)$ & 4.33 & 6.06 & $5.19(163)$ \\
\hline 4 & 60.02 & 70.42 & $65.22(197)$ & 5.81 & 4.66 & $6.51(175)$ \\
\hline LSD1\% & 0.21 & 0.27 & 0.25 & 0.06 & 0.8 & 0.07 \\
\hline
\end{tabular}

E1-4: O control, phenylalanine yeast and coubled phenylalanine and yeast, respectively; F1-4 NPK chemical, traditional fertilizer, 1/2NPK + PGPB, humic acid + PGPB, and moringa dry leaves extract + PGPB respectivel. 
Table 4. Pooled mean (2018, 2019 seasons) for major phenolic acids, rosmarynic acid (RAC) and carnosic acid (CSC) under E1-4 interacted with F1-4 for R-officinals.

\begin{tabular}{ccccccc}
\hline $\begin{array}{c}\text { Application } \\
\text { Treatment }\end{array}$ & \multicolumn{3}{c}{ RAC } & & & \multicolumn{2}{c}{ CTC } \\
\cline { 2 - 7 } & $\mathbf{2 0 1 8}$ & $\mathbf{2 0 1 9}$ & Pooled mean & $\mathbf{2 0 1 8}$ & $\mathbf{2 0 1 9}$ & Pooled mean \\
\hline $\mathrm{F}_{1} \mathrm{E}_{1}$ & 16.51 & 13.62 & $15.06(100)$ & 112.15 & 95.74 & $103.94(100)$ \\
2 & 20.63 & 17.02 & $18.82(125)$ & 159.25 & 135.95 & $147.60(142)$ \\
3 & 21.95 & 18.11 & $20.03(133)$ & 168.22 & 143.61 & $155.91(150)$ \\
4 & 23.11 & 19.06 & $21.08(140)$ & 188.41 & 160.84 & $174.62(168)$ \\
\hline $\mathrm{F}_{2} \mathrm{E}_{1}$ & 17.83 & 14.70 & $16.26(108)$ & 128.97 & 110.10 & $119.53(115)$ \\
2 & 21.79 & 17.97 & $19.88(132)$ & 177.19 & 151.26 & $164.22(158)$ \\
3 & 23.93 & 19.74 & $21.83(145)$ & 185.04 & 157.97 & $171.50(165)$ \\
4 & 25.92 & 21.38 & $23.65(157)$ & 192.89 & 164.67 & $178.78(172)$ \\
\hline $\mathrm{F}_{3} \mathrm{E}_{1}$ & 18.49 & 15.26 & $16.87(112)$ & 143.55 & 122.54 & $133.04(128)$ \\
2 & 23.11 & 19.06 & $21.08(140)$ & 185.04 & 157.97 & $171.50(165)$ \\
3 & 24.93 & 20.56 & $22.74(151)$ & 190.65 & 162.75 & $176.70(170)$ \\
4 & 27.24 & 22.47 & $24.84(165)$ & 202.99 & 173.65 & $188.32(181)$ \\
\hline $\mathrm{F}_{4} \mathrm{E}_{1}$ & 18.99 & 15.66 & $17.32(115)$ & 148.03 & 126.37 & $137.20(132)$ \\
2 & 25.09 & 20.70 & $22.89(152)$ & 194.01 & 165.63 & $179.82(173)$ \\
3 & 27.41 & 22.61 & $25.01(166)$ & 207.47 & 177.11 & $192.29(185)$ \\
4 & 29.38 & 24.24 & $26.8(178)$ & 216.44 & 184.77 & $200.40(193)$ \\
\hline LSD1\% & 0.17 & 0.12 & 0.15 & 0.85 & 0.72 & 0.81 \\
\hline
\end{tabular}

E1-4: O control, phenylalanine), yeast and coubled phenylalanine and yeast, respectively; F1-4 NPK chemical, traditional fertilizer, 1/2NPK + PGPB, humic acid + PGPB, and moringa dry leaves extract + PGPB, respectively.

Table 5. Essential oil contents for pooled mean (2018, 2019 seasons) for R-officinals under F1-4 interacted with E1-4.

\begin{tabular}{|c|c|c|c|c|c|c|c|c|c|c|c|}
\hline \multirow{2}{*}{$\begin{array}{c}\text { Application } \\
\text { Treatment }\end{array}$} & \multicolumn{11}{|c|}{ EO components } \\
\hline & a-Penine & Comphere & Limonine & Cineol & Terpinene & p-cymene & Linlool & Terpineol & Caryophllene a & $\alpha$-Terpineol & Total \\
\hline $\mathrm{F}_{1} \mathrm{E}_{1}$ & 15.50 & 5.80 & 3.22 & 20.33 & 1.12 & 2.42 & 16.57 & 1.51 & 1.70 & 2.82 & 70.99 \\
\hline 2 & 16.12 & 6.03 & 3.35 & 21.14 & 1.16 & 2.52 & 17.23 & 1.57 & 1.77 & 2.93 & 73.82 \\
\hline 3 & 16.59 & 6.21 & 3.45 & 21.75 & 1.20 & 2.59 & 18.05 & 1.62 & 1.82 & 3.02 & 76.30 \\
\hline 4 & 16.90 & 6.32 & 3.51 & 22.16 & 1.31 & 2.64 & 18.06 & 1.65 & 1.85 & 3.07 & 77.47 \\
\hline $\mathrm{F}_{2} \mathrm{E}_{1}$ & 15.97 & 5.97 & 3.32 & 20.94 & 1.15 & 2.49 & 17.07 & 1.56 & 1.75 & 2.90 & 76.76 \\
\hline 2 & 16.43 & 6.15 & 3.41 & 21.55 & 1.19 & 2.57 & 17.56 & 1.60 & 1.76 & 2.99 & 75.21 \\
\hline 3 & 16.74 & 6.26 & 3.48 & 21.96 & 1.21 & 2.61 & 17.90 & 1.63 & 1.82 & 3.05 & 76.66 \\
\hline 4 & 17.52 & 6.55 & 3.64 & 22.97 & 1.27 & 2.74 & 18.72 & 1.71 & 1.85 & 3.19 & 80.16 \\
\hline $\mathrm{F}_{3} \mathrm{E}_{1}$ & 16.28 & 6.09 & 3.38 & 21.35 & 1.18 & 2.54 & 17.40 & 1.59 & 1.79 & 2.96 & 74.56 \\
\hline 2 & 17.05 & 6.38 & 3.54 & 22.36 & 1.23 & 2.66 & 18.23 & 1.66 & 1.87 & 3.10 & 78.08 \\
\hline 3 & 17.36 & 6.50 & 3.61 & 22.77 & 1.26 & 2.71 & 18.56 & 1.69 & 1.90 & 3.16 & 79.52 \\
\hline 4 & 17.83 & 6.67 & 3.70 & 23.38 & 1.29 & 2.78 & 19.06 & 1.74 & 1.96 & 3.24 & 81.65 \\
\hline $\mathrm{F}_{4} \mathrm{E} 1$ & 16.59 & 6.21 & 3.45 & 21.75 & 1.20 & 2.59 & 17.73 & 1.62 & 1.82 & 3.02 & 75.98 \\
\hline 2 & 17.98 & 6.73 & 3.74 & 23.58 & 1.30 & 2.81 & 19.22 & 1.75 & 1.97 & 3.27 & 82.35 \\
\hline 3 & 18.29 & 6.84 & 3.80 & 23.99 & 1.32 & 2.86 & 19.55 & 1.78 & 2.01 & 3.33 & 83.77 \\
\hline 4 & 18.91 & 7.08 & 3.93 & 24.80 & 1.37 & 2.95 & 20.22 & 1.84 & 2.07 & 3.44 & 86.61 \\
\hline LSD $1 \%$ & & & & & & & & & & & 0.42 \\
\hline
\end{tabular}

E1-4: O control, phenylalanine yeast and coubled phenylalanine and yeast, respectively; F1-4 NPK chemical, traditional fertilizer, 1/2NPK + PGPB, humic acid + PGPB, and moringa dry leaves extract + PGPB, respectively. 


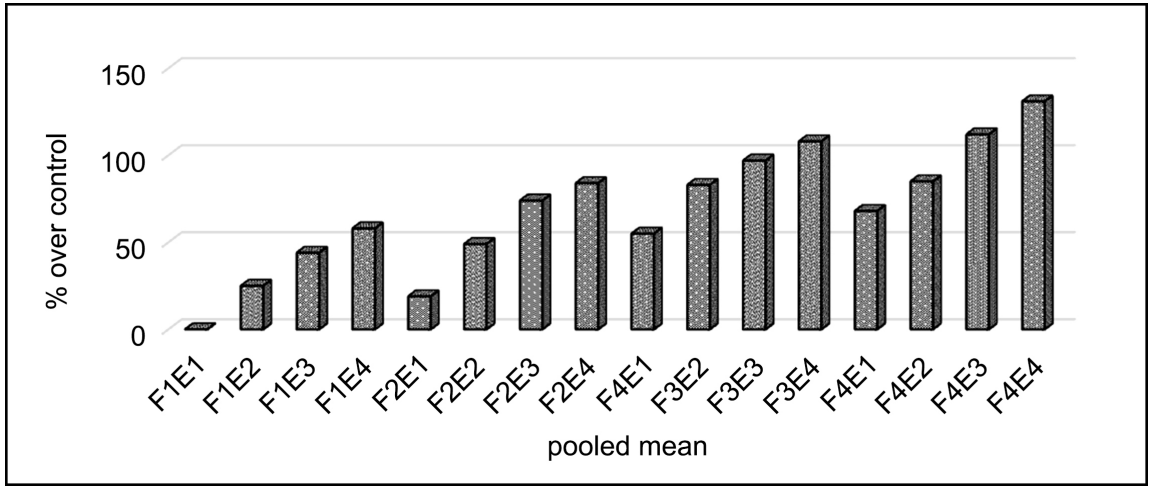

Figure 2. Pooled mean EOY, $\mathrm{g} / \mathrm{m}^{2}$ as $\%$ over control under E1-4 interacted with F1-4 for R. officinals.

\section{Conclusion}

According to our study it declared multi-repeating elicitation with yeast $\left(\mathrm{E}_{3}\right)$, phenylolanine $\left(\mathrm{E}_{2}\right)$ and coupled $\left(\mathrm{E}_{2+3}\right)$ under organic system, bio fertilizers (humic acid + PGPB, $\mathrm{F}_{3}$; Moringa dry leaves extract + PGPB, $\mathrm{F}_{4}$ ) ensured sustainable development and sustainable reliable practical application, without NPK system (F1), While F2 reduced NPK, application.

\section{Conflicts of Interest}

The authors declare no conflicts of interest regarding the publication of this paper.

\section{References}

[1] Gharib, F., Ghazi, S., Aly, H., EL-Araby, M. and Mousstafa, S. (2016) Effect of Soil Type and Water Content on Rosemary Growth and Essential Oil Yield. International Journal of Scientific and Engineering Research, 7, 183-189.

[2] González-Trujano, M.E., Peña, E.I., Martínez, A.L., Moreno, J., Guevara-Fefer, P., Déciga-Campos, M., et al. (2007) Evaluation of the Antinociceptive Effect of Rosmarinus officinalis L. Using Three Different Experimental Models in Rodents. Journal of Ethnopharmacology, 111, 476-482.

https://doi.org/10.1016/j.jep.2006.12.011

[3] Pintore, G., Usai, M., Bradesi, P., Juliano, C., Boatto, G., Tomi, F., Chessa, M., Cerri, R. and Casanova, J. (2002) Chemical Composition and Antimicrobial Activity of Rosmarinus officinalis L. Oils from Sardinia and Corsica. Flavour and Fragrance Journal, 17, 15-19. https://doi.org/10.1002/ffj.1022

[4] Yu, M.H., Choi, J.H., Chae, I.G., Im, H.G., Yang, S.A., More, K., Lee, I.S. and Lee, J. (2013) Suppression of LPS-Induced Inflammatory Activities by Rosmarinus officinalis L. Food Chemistry, 136, 1047-1054. https://doi.org/10.1016/j.foodchem.2012.08.085

[5] Raskovic, A., Milanovic, I., Pavlovic, N., Cebovic, T., Vukmirovic, S. and Mikov, M. (2014) Antioxidant Activity of Rosemary (Rosmarinus officinalis L.) Essential Oil and Its Hepatoprotective Potential. BMC Complementary and Alternative Medicine, 14, Article No. 225. https://doi.org/10.1186/1472-6882-14-225

[6] Abu-Al-Basal, M.A. (2010) Healing Potential of Rosmarinus officinalis L. on Full-Thickness Excision Cutaneous Wounds in Alloxan-Induced-Diabetic BALB/c 
Mice. Journal of Ethnopharmacology, 131, 443-450.

https://doi.org/10.1016/j.jep.2010.07.007

[7] Yadav, E., Kumar, S., Mahant, S., Khatkar, S. and Rao, R. (2017) Tea Tree Oil: A Promising Essential Oil. Journal of Essential Oil Research, 29, 201-213. https://doi.org/10.1080/10412905.2016.1232665

[8] deMedeiros Barbosa, I., da Costa Medeiros, J.A., de Oliveira, K.A.R., Gomes-Neto, N.J., Tavares, J.F., Magnani, M. and de Souza, E.L. (2016) Efficacy of the Combined Application of Oregano and Rosemary Essential Oils for the Control of Escherichia coli, Listeria monocytogenes and Salmonella enteritidis in Leafy Vegetables. Food Control, 59, 468-477. https://doi.org/10.1016/j.foodcont.2015.06.017

[9] Saporito, F., Sandri, G., Bonferoni, M.C., Rossi, S., Boselli, C., Icaro, C.A., et al. (2018) Essential Oil-Loaded Lipid Nanoparticles for Wound Healing. International Journal of Nanomedicine, 13, 175-186. https://doi.org/10.2147/IJN.S152529

[10] Daferera, D.J., Ziogas, B.N. and Polissiou, M.G. (2000) GC-MS Analysis of Essential Oils from Some Greek Aromatic Plants and Their Fungitoxicity on Penicillium digitatum. Journal of Agricultural and Food Chemistry, 48, 2576-2581. https://doi.org/10.1021/jf990835x

[11] Bozin, B., Mimica-Dukic, N., Samojlik, I. and Jovin, E. (2007) Antimicrobial and Antioxidant Properties of Rosemary and Sage (Rosmarinus officinalis L. and Salvia officinalis L., Lamiaceae) Essential Oils. Journal of Agricultural and Food Chemistry, 55, 7879-7885. https://doi.org/10.1021/jf0715323

[12] El-Omri, A., Junkyu, H., Parida, Y., Kiyokazu, K., Ben Abdrabbah, M. and Hiroko, I. (2010) Rosmarinus officinalis Polyphenols Activate Cholinergic Activities in PC12 Cells through Phosphorylation of ERK1/2. Journal of Ethnopharmacology, 131, 451-458. https://doi.org/10.1016/j.jep.2010.07.006

[13] Wada, M., Kido, H., Ohyama, K., Kishikawa, N., Ohba, Y., Kuroda, N. and Naka, S.K. (2004) Evaluation of Quenching Effects of Non-Water-Soluble and Water-Soluble Rosemary Extracts against Active Oxygen Species by Chemiluminescent Assay. Food Chemistry, 87, 261-267. https://doi.org/10.1016/j.foodchem.2003.11.017

[14] Cuvelier, M.-E., Richard, H. and Berest, C. (1996) Antioxidative Activity and Phenolic Composition of Pilot-Plant and Commercial Extracts of Sage and Rosemary. Journal of the American Oil Chemists' Society, 73, 645-652. https://doi.org/10.1007/BF02518121

[15] Scheckel, K.A., Degner, S.C. and Romagnolo, D.F. (2008) Rosmarinic Acid Antagonizes Activator Protein-1-Dependent Activation of Cyclooxygenase-2 Expression in Human Cancer and Nonmalignant Cell Lines. The Journal of Nutrition, 138, 2098-2105. https://doi.org/10.3945/jn.108.090431

[16] Karthik, D., Viswanathan, P. and Anuradha, C.V. (2011) Administration of Rosmarinic acid Reduces Cardiopathology and Blood Pressure through Inhibition of p22phox NADPH Oxidase in Fructose-Fed Hypertensive Rats. Journal of Cardiovascular Pharmacology, 58, 514-521.

[17] Fernández, L.F., Palomino, O.M. and Frutos, G. (2014) Effectiveness of Rosmarinusofficinalis Essential Oil as Antihypotensive Agent in Primary Hypotensive Patients and Its Influence on Health-Related Quality of Life. Journal of Ethnopharmacology, 151, 509-516.

[18] Amaral, G.P., de Carvalho, N.R., Barcelos, R.P., Dobrachinski, F., PortellaRde, L., da Silva, M.H., et al. (2013) Protective Action of Ethanolic Extract of Rosmarinusoffi- 
cinalis L. in Gastric Ulcer Prevention Induced by Ethanol in Rats. Food and Chemical Toxicology, 55, 48-55.

[19] Bosadas, S.J., Caz, V., Largo, C., De La Gandare, B., Matallanas, B., Reglero, G., et al. (2009) Protective Effect of Supercritical Fluid Rosemary Extract, Rosmarinus officinalis, on Antioxidants of Major Organs of Aged Rats. Experimental Gerontology, 44, 383-389. https://doi.org/10.1016/j.exger.2009.02.015

[20] Kayashima, T. and Matsubara, K. (2012) Antiangiogenic Effect of Carnosic Acid and Carnosol, Neuroprotective Compounds in Rosemary Leaves. Bioscience, Biotechnology, and Biochemistry, 76, 115-119. https://doi.org/10.1271/bbb.110584

[21] Sinkovic, A., Suran, D., Lokar, L., Fliser, E., Skerget, M., Novak, Z., et al. (2011) Rosemary Extracts Improve Flow-Mediated Dilatation of the Brachial Artery and Plasma PAI-1 Activity in Healthy Young Volunteers. Phytotherapy Research, 25, 402-407. https://doi.org/10.1002/ptr.3276

[22] Yesil-Celiktas, O., Sevimli, C., Bedir, E. and Vardar-Sukan, F. (2010) Inhibitory Effects of Rosemary Extracts, Carnosic Acid and Rosmarinic Acid on the Growth of Various Human Cancer Cell Lines. Plant Foods for Human Nutrition, 65, 158-163. https://doi.org/10.1007/s11130-010-0166-4

[23] Menghini, L., Genovese, S., Epifano, F., Tirillini, B., Ferrante, C. and Leporini, L. (2010) Antiproliferative, Protective and Antioxidant Effects of Artichoke, Dandelion, Turmeric and Rosemary Extracts and Their Formulation. International Journal of Immunopathology and Pharmacology, 23, 601-610.

https://doi.org/10.1177/039463201002300222

[24] Kontogianni, V.G., Tomic, G., Nikolic, I., Nerantzaki, A.A., Sayyad, N., Stosic-Grujicic, S., et al. (2013) Phytochemical Profile of Rosmarinus officinalis and Salvia officinalis Extracts and Correlation to Their Antioxidant and Anti-Proliferative Activity. Food Chemistry, 136, 120-129. https://doi.org/10.1016/j.foodchem.2012.07.091

[25] Nolkemper, S., Reichling, J., Stintzing, F.C., Carle, R. and Schnitzler, P. (2006) Antiviral Effect of Aqueous Extracts from Species of the Lamiaceae Family against Herpes Simplex Virus Type 1 and Type 2 in Vitro. Planta Medica, 72, 1378-1382. https://doi.org/10.1055/s-2006-951719

[26] Bernardes, W.A., Lucarini, R., Tozatti, M.G., Flauzino, L.G., Souza, M.G., Turatti, I.C., et al. (2010) Antibacterial Activity of the Essential Oil from Rosmarinus officinalis and Its Major Components against Oral Pathogens. Zeitschrift fur Naturforschung, 65, 588-593. https://doi.org/10.1515/znc-2010-9-1009

[27] Ramadan, K.S., Khalil, O.A., Danial, E.N., Alnahdi, H.S. and Ayaz, N.O. (2013) Hypoglycemic and Hepatoprotective Activity of Rosmarinus officinalis Extract in Diabetic Rats. The Journal of Physiology and Biochemistry, 69, 779-783.

https://doi.org/10.1007/s13105-013-0253-8

[28] El Saied Azab, A., Fetouh, F.A. and Albasha, M.O. (2014) Nephro-Protective Effects of Curcumin, Rosemary and Propolis against Gentamicin Induced Toxicity in Guinea Pigs: Morphological and Biochemical Study. American Journal of Clinical and Experimental Medicine, 2, 28-35. https://doi.org/10.11648/j.ajcem.20140202.14

[29] Del Baño, M.J., Castillo, J., Benavente-García, O., Lorente, J., Martín-Gil, R., Acevedo, C., et al. (2006) Radioprotective-Antimutagenic Effects of Rosemary Phenolics against Chromosomal Damage Induced in Human Lymphocytes by $\gamma$-Rays. Journal of Agricultural and Food Chemistry, 54, 2064-2068.

https://doi.org/10.1021/jf0581574

[30] al-Hader, A.A., Hasan, Z.A. and Aqel, M.B. (1994) Hyperglycemic and Insulin Re- 
lease Inhibitory Effects of Rosmarinus officinalis. Journal of Ethnopharmacology, 43, 217-221. https://doi.org/10.1016/0378-8741(94)90046-9

[31] Tabassum, N. and Hamdani, M. (2014) Plants Used to Treat Skin Diseases. Pharmacognosy Reviews, 8, 52-60. https://doi.org/10.4103/0973-7847.125531

[32] Machado, D.G., Cunha, M.P., Neis, V.B., Balen, G.O., Colla, A.R., Grando, J., et al. (2012) Rosmarinus officinalis L. Hydroalcoholic Extract, Similar to Fluoxetine, Reverses Depressive-Like Behavior without Altering Learning Deficit in Olfactory Bulbectomized Mice. Journal of Ethnopharmacology, 143, 158-169. https://doi.org/10.1016/j.jep.2012.06.017

[33] Nie, J.-Y., Li, R., Wang, Y., Tan, J., Tang, S.-H. and Jiang, Z.-T. (2019) Antioxidant Activity Evaluation of Rosemary Ethanol Extract and Their Cellular Antioxidant Activity toward HeLa Cells. Journal of Food Biochemistry, 43, e12851. https://doi.org/10.1111/jfbc.12851

[34] Petersen, M. and Simmonds, M.S. (2003) Rosmarinic Acid. Phytochemistry, 62, 121-125. https://doi.org/10.1016/S0031-9422(02)00513-7

[35] Romano, C.S., Abadi, K., Repetto, V., Vojnov, A.A. and Moreno, S. (2009) Synergistic Antioxidant and Antibacterial Activity of Rosemary plus Butylated Derivatives. Food Chemistry, 115, 456-461. https://doi.org/10.1016/j.foodchem.2008.12.029

[36] Sánchez-Camargo, A.d.P., Valdés, A., Sullini, G., García-Cañas, V., Cifuentes, A., Ibáñez, E. and Herrero, M. (2014) Two-Step Sequential Supercritical Fluid Extracts from Rosemary with Enhanced Anti-Proliferative Activity. Journal of Functional Foods, 11, 293-303. https://doi.org/10.1016/j.jff.2014.10.014

[37] Moore, J., Yousef, M. and Tsiani, E. (2016) Anticancer Effects of Rosemary (Rosmarinus officinalis L.) Extract and Rosemary Extract Polyphenols. Nutrients, 8, 731. https://doi.org/10.3390/nu8110731

[38] Aruoma, O.I., Spencer, J.P.E., Rossi, R., Aeschbach, R., Khan, A., Mahmood, N., Munoz, A., Murcia, A., Butler, J. and Halliwell, B. (1996) An Evaluation of the Antioxidant and Antiviral Action of Extracts of Rosemary and Provençal Herbs. Food and Chemical Toxicology, 34, 449-456.

https://doi.org/10.1016/0278-6915(96)00004-X

[39] Kashiwada, Y., Nagao, T., Hashimoto, A., Ikeshiro, Y., Okabe, H., Cosentino, L.M. and Lee, K.H. (2000) Anti-AIDS Agents 38. Anti-HIV Activity of 3-O-Acyl Ursolic Acid Derivatives. Journal of Natural Products, 63, 1619-1622.

https://doi.org/10.1021/np990633v

[40] Satoh, T., Kosaka, K., Itoh, K., Kobayashi, A., Yamamoto, M., Shimojo, Y., Kitajima, C., Cui, J., Kamins, J., Okamoto, S., et al. (2008) Carnosic Acid, a Catechol-Type Electrophilic Compound, Protects Neurons Both in Vitro and in Vivo through Activation of the Keap1/Nrf2 Pathway via $S$-Alkylation of Targeted Cysteines on Keap1. Journal of Neurochemistry, 104, 1116-1131. https://doi.org/10.1111/j.1471-4159.2007.05039.x

[41] Fischedick, J.T., Standiford, M., Johnson, D.A. and Johnson, J.A. (2013) Structure Activity Relationship of Phenolic Diterpenes from Salvia officinalis as Activators of the Nuclear Factor E2-Related Factor 2 Pathway. Bioorganic \& Medicinal Chemistry Letters, 21, 2618-2622. https://doi.org/10.1016/j.bmc.2013.02.019

[42] Rasoulian, B., Hajializadeh, Z., Esmaeili-Mahani, S., Rashidipour, M., Fatemi, I. and Kaeidi, A. (2018) Neuroprotective and Antinociceptive Effects of Rosemary (Rosmarinus officinalis L.) Extract in Rats with Painful Diabetic Neuropathy. The Journal of Physiological Sciences, 69, 57-64. https://doi.org/10.1007/s12576-018-0620-x 
[43] Frankel, E.N., Huang, S.-W., Aescbbach, R. and Prior, E. (1996) Antioxidant Activity of a Rosemary Extract and Its Constituents, Carnosic Acid, Carnosol, and Rosmarinic Acid, in Bulk Oil and Oil-in-Water Emulsion. Journal of Agricultural and Food Chemistry, 44, 131-135. https://doi.org/10.1021/jf950374p

[44] Moreno, S., Seheyer, T., Romano, C.S. and Vojnov, A.A. (2006) Antioxidant and Antimicrobial Activities of Rosemary Extracts Linked to Their Polyphenol Composition. Free Radical Research, 40, 223-231.

https://doi.org/10.1080/10715760500473834

[45] Costa, S., Utan, A., Speroni, F., Cervellatti, R., Piva, G., Prandini, A., et al. (2007) Carnosic Acid from Rosemary Extracts: A Potential Chemoprotective Agent against Aflatoxin B1. An in Vitro Study. Journal of Applied Toxicology, 27, 152-159. https://doi.org/10.1002/jat.1186

[46] Estevez, M. and Cava, R. (2006) Effectiveness of Rosemary Essential Oil as an Inhibitor of Lipid and Protein Oxidation: Contradictory Effects in Different Types of Frankfurters. Meat Science, 72, 348-355.

https://doi.org/10.1016/j.meatsci.2005.08.005

[47] Houlihan, C.M., Ho, C.T. and Chang, S.S. (1985) The Structure of Rosmariquinone-A New Antioxidant Isolated from Rosmarinus officinalis L. Journal of the American Oil Chemists' Society, 62, 96-98. https://doi.org/10.1007/BF02541500

[48] Ahsaei, S.M., Rodriguez-Roijo, S., Salgado, M., Coccero, M.J., Talebi-Jahromi, K. and Amoabedin, G. (2020) Insecticidal Activity of Spray Dried Microencapsulated Essential Oils of Rosmarinus officinalis and Zataria multiflora against Tribolium confusum. Crop Protection, 128, Article ID: 104996.

https://doi.org/10.1016/j.cropro.2019.104996

[49] Elgharabawy, R.M., Aldubayan, M., Alshaifani, M.A. and Ahmed, A.S. (2020) Beneficial Role of Rosemary Aqueous Extracts against Boldenone Induced Cardiac Toxicity, Injury and Oxidative Stress, in Male Rats. International Journal of Pharmacology, 16, 136-144. https://doi.org/10.3923/ijp.2020.136.144

[50] Bahri, S., Ben Ali, R., Abdennabi, R., Ben Said, D., Mlika, M., Ben Fradj, M.K. and El-May, M.V. (2020) Comparison of the Protective Effect of Salvia officinalis and Rosmarinus officinalis Infusions against Hepatic Damage Induced by Hypotermic-Ischemia in Wistar Rats. Nutrition and Cancer, 72, 283-292.

https://doi.org/10.1080/01635581.2019.1631359

[51] Leja, K., Majcher, M., Juzwa, W., Czaczyk, K. and Komosa, M. (2020) Comparative Evaluation of Piper nigrum, Rosmarinus officinalis, Cymbopogon citratus and Juniperus communis L. Essential Oils of Different Origin as Functional Antimicrobials in Foods. Food, 9, 141. https://doi.org/10.3390/foods9020141

[52] Angelova, Z., Gergiev, S. and Roes, W. (2006) Elicitation of Plants. Biotechnology \& Biotechnological Equipment, 20, 72-83.

https://doi.org/10.1080/13102818.2006.10817345

[53] Namdeo, A.G. (2007) Plant Cell Elicitation for Production of Secondary Metabolites: A Review. Pharmacognosy Reviews, 1, 69-79.

[54] Świeca, M. (2016) Elicitation and Treatment with Precursors of Phenolics Synthesis Improve Low-Molecular Antioxidants and Antioxidant Capacity of Buckwheat Sprouts. Acta Scientiarum Polonorum Technologia Alimentaria, 15, 17-28. https://doi.org/10.17306/J.AFS.2016.1.2

[55] Sharma, R.Z. (2016) Optimization of Methyl Jasmonate and $\beta$-Cyclodextrin for Enhanced Production of Taraxerol and Taraxasterol in (Taraxacum officinale Weber) Cultures. Plant Physiology and Biochemistry, 103, 24-30. 
https://doi.org/10.1016/j.plaphy.2016.02.029

[56] Mohammadparast, B., Rasoul, M., Rustaiee, A.R., Zardari, S. and Agrawal, V. (2014) Quantification of Asiatic Acid from Plant Parts of Centella asiatica L. and Enhancement of Its Synthesis through Organic Elicitors in in vitro. Horticulture, Environment, and Biotechnology, 55, 578-582. https://doi.org/10.1007/s13580-014-0168-5

[57] Mejdoub-Trabelsi, B., Touihri, S., Ammar, N., Riahi, A. and Daami-Remadi, M. (2020) Effect of Chitosan for the Control of Potato Diseases Caused by Fusarium Species. Journal of Phytopathology, 168, 18-27. https://doi.org/10.1111/jph.12847

[58] Ahmad, Z., Khan, Q.U., Qadoos, A., Khan, M.J., Saleem, A. and Bibi, Z. (2020) Humic Acid, an Effective Amendment Used for Amelioration of Phosphatic Fertilizer and Enhancing Maize Yield. Pure and Applied Biology, 9, 750-759. https://doi.org/10.19045/bspab.2020.90081

[59] Gao, M.B., Zhang, W. and Ruan, C.-J. (2011) Significantly Improved Taxuyunnanine C Production in Cell Suspension Cultures of Taxus chinensis by Process Intensification of Repeated Elicitation, Sucrose Feeding, and in Situ Adsorption. World Journal of Microbiology and Biotechnology, 27, 2271-2279. https://doi.org/10.1007/s11274-011-0690-x

[60] Wang, Y.N., Wei, Y.H., Hao, H.Y., et al. (2007) Advances in the Research of Resveratrol Metabolins. Acta Botanica Boreali-Occidentalia Sinica, 27, 852-857

[61] Jansen, M.A.K., Hectors, K., O’Brien, N.M., Guisez, Y. and Potters, G. (2008) Plant Stress and Human Health: Do Human Consumers Benefit from UV-B Acclimated Crops? Plant Science, 175, 449-458. https://doi.org/10.1016/j.plantsci.2008.04.010

[62] Treutter, D. (2005) Significance of Flavonoids in Plant Resistance and Enhancement of Their Biosynthesis. Plant Biology, 7, 581-591.

https://doi.org/10.1055/s-2005-873009

[63] Sharifi-Rad, J., Sharifi-Rad, M. and Teixeira da Silva, J.A. (2016) Morphological, Physiological and Biochemical Responses of Crops (Zea mays L., Phaseolus vulgaris L.), Medicinal Plants (Hyssopus officinalis L., Nigella sativa L.), and Weeds (Amaranthus retroflexus L., Taraxacum officinale F. H. Wigg) Exposed to $\mathrm{SiO}_{2}$ Nanoparticles. Journal of Agricultural Science and Technology, 18, 1027-1040.

[64] Jalil Sheshbahreh, M., Movahhedi Dehnavi, M., Salehi, A. and Bahreininejad, B. (2019) Effect of Irrigation Regimes and Nitrogen Sources on Biomass Production, Water and Nitrogen Use Efficiency and Nutrients Uptake in Coneflower (Echinacea purpurea L.). Agricultural Water Management, 213, 358-367. https://doi.org/10.1016/j.agwat.2018.10.011

[65] Złotek, U. (2017) Affect of Jasmonic Acid and Yeast Extract Elicitation on Low-Molecular Antioxidants and Antioxidant Activity of Marjoram (Origanum marjoram L). Acta Scientiarum Polonorum Technologia Alimentaria, 16, 371-377. https://doi.org/10.17306/J.AFS.2017.0505

[66] Pereira, L.A. and Raimunda, A.D.S. (2016) The Intensive Use of Pesticides-The New Face of the Agrarian Question. OKARA: Geografia em Debate, 10, 185-194.

[67] Zheng, L., Hong, F.S., Lu, S.P. and Liu, C. (2005) Effect of $\mathrm{Nano}^{-\mathrm{TiO}_{2}}$ on Strength of Naturally Aged Seeds and Growth of Spinach. Biological Trace Element Research, 104, 83-91. https://doi.org/10.1385/BTER:104:1:083

[68] Nanda, A.K., Andrio, E., Marino, D., Pauly, N. and Dunand, C. (2010) Reactive Oxygen Species during Plant-Microorganism Early Interactions. Journal of Integrative Plant Biology, 52, 195-204. https://doi.org/10.1111/j.1744-7909.2010.00933.x 
[69] Adam, A., Jourdan, E., Pgena, M., Duby, F., Domes, J. and Thonart, P. (2005) Resistance Induced in Cucumber and Tomato by a Non-Pathogenic Pseudomonas putida Strain. Parasitica, 6, 13-22.

[70] Gabaston, J., EL-Khawand, T., Waffo-Teguo, P., Decendit, A., Richard, T., Merillon, J.M. and Pavela, R. (2018) Stilbenes from Grapevine Root: A Promising Natural Insecticide against Leptinotarsa decemlineata. Journal of Pest Science, 91, 897-906. https://doi.org/10.1007/s10340-018-0956-2

[71] Hasan, M. and Bae, H.H. (2017) An Overview of Stress-Induced Resveratrol Synthesis in Grapes: Perspectives for Resveratrol-Enriched Grape Products. Molecules, 22, 294. https://doi.org/10.3390/molecules22020294

[72] Lazzari, F.M. and Souza, A.S. (2017) Green Revolution: Impacts on Traditional Knowledge. 4o Congresso Internacional de Direito e Contemporaneidade, 1-16.

[73] Matos, P.F. and Pessoa, V.L.S. (2011) The Modernization of Agriculture in Brazil and the New Uses of the Territory. Geo UERJ, 2, 290-322.

https://doi.org/10.12957/geouerj.2011.2456

[74] Brasil. Ministério de Agricultura, Pecuária e Abastecimento. News: In 7 Years, It Triples the Number of Organic Producers Registered in the Ministry.

https://www.gov.br/noticias/em-sete-anos-triplica-o-numero-de-produtoresorganic os-\%20cadastrados-no-mapa\%3e

[75] Bombardi, L.M. (2019) Geografia do Uso de Agrotóxicos no Brasil e Conexões com a União Europeia. 2nd Edition, FFLCH-USP, São Paulo.

[76] Nasrala Neto, E., et al. (2014) Health Surveillance and Agribusiness: The Impact of Pesticides on Health and the Environment. Danger Ahead! Ciência \& Saúde Coletiva, 19, 4709-4718. https://doi.org/10.1590/1413-812320141912.03172013

[77] Rattner, H. and Franco Netto, G. (2009) Environment, Health and Sustainable Development. Ciência \& Saúde Coletiva, 14, 1965-1971. https://doi.org/10.1590/S1413-81232009000600002

[78] Pignati, W.A., et al. (2017) Distribuição espacial do uso de agrotóxicos no Brasil: Uma ferramenta para a Vigilância em Saúde. Ciência \& Saúde Coletiva, 22, 3281-3293. https://doi.org/10.1590/1413-812320172210.17742017

[79] de Abreu, P.H.B. and Alonzo, H.G.A. (2014) Rural Work and Health Risks: A Review into de "Safe Use" of Pesticides in Brazil. Ciência \& Saúde Coletiva, 19, 4197-4208. https://doi.org/10.1590/1413-812320141910.09342014

[80] Cong, T., Ristaino, J.B. and Hu, S. (2006) Soil Microbial Biomass and Activity in Organic Tomato Farming Systems: Effects of Organic Inputs and Straw Mulching. Soil Biology and Biochemistry, 38, 247-255.

https://doi.org/10.1016/j.soilbio.2005.05.002

[81] Vogl, C.R., Kilcher, L. and Schmidt, H. (2005) Are Standards and Regulations of Organic Farming Moving Away from Small Farmers' Knowledge? Journal of Sustainable Agriculture, 26, 5-26. https://doi.org/10.1300/J064v26n01_03

[82] FiBL (2019). Global Organic Area Reaches Another All-Time High. Media Release, 13 February 2019.

[83] Reganold, J.P. and Wachter, J.M. (2016) Organic Agriculture in the Twenty-First Century. Nature Plants, 2, Article No. 15221. https://doi.org/10.1038/nplants.2015.221

[84] Hussein, M., El-Sherbeny, S., Khalil, M., Naguib, N. and Aly, S. (2006) Growth Characters and Chemical Constituents of Dracocephalum moldavica L. Plants in Relation to Compost Fertilizer and Planting Distance. Scientia Horticulturae, 108, 
322-331. https://doi.org/10.1016/j.scienta.2006.01.035

[85] Mohammadi, H., Dashi, R., Farzaneh, M., Parviz, L. and Hashempour, H. (2017) Effects of Beneficial Root Pseudomonas on Morphological, Physiological, and Phytochemical Characteristics of Satureja hortensis (Lamiaceae) under Water Stress. Brazilian Journal of Botany, 40, 41-48. https://doi.org/10.1007/s40415-016-0319-2

[86] Banchio, E., Bogino, P.C., Zygadlo, J. and Giordano, W. (2008) Plant Growth Promoting Rhizobacteria Improve Growth and Essential Oil Yield in Origanum majorana L. Biochemical Systematics and Ecology, 36, 766-771. https://doi.org/10.1016/j.bse.2008.08.006

[87] Cappellari, L.R., Santoro, M.V., Nievas, F., Giordano, W. and Banchio, E. (2013) Increase of Secondary Metabolite Content in Marigold by Inoculation with Plant Growth-Promoting Rhizobacteria. Applied Soil Ecology, 70, 16-22. https://doi.org/10.1016/j.apsoil.2013.04.001

[88] Zhao, Q., Wu, Y.N., Fan, Q., Han, Q.Q., Paré, P.W., Xu, R., et al. (2016) Improved Growth and Metabolite Accumulation in Codonopsis pilosula (Franch.) Nannf. by Inoculation of Bacillus amyloliquefaciens GB03. Journal of Agricultural and Food Chemistry, 64, 8103-8108. https://doi.org/10.1021/acs.jafc.6b03390

[89] Damam, M., Kaloori, K., Gaddam, B. and Kausar, R. (2016) Plant Growth Promoting Substances (Phytohormones) Produced by Rhizobacterial Strains Isolated from the Rhizosphere of Medicinal Plants. International Journal of Pharmaceutical Sciences Review and Research, 37, 130-136.

[90] Yadav, K.K. and Sarkar, S. (2019) Biofertilizers, Impact on Soil Fertility and Crop Productivity under Sustainable Agriculture. Environment and Ecology, 37, 89-93.

[91] Panya, M., Naresh Kumar, G. and Rajkumar, S. (2013) Invasion of Rhizobial Infection Thread by Non-Rhizobia for Colonization of Vigna radiata Root Nodules. FEMS Microbiology Letters, 348, 58-65. https://doi.org/10.1111/1574-6968.12245

[92] Chagas, F.O., Pessotti, R.D.C., Caraballo-Rodríguez, A.M. and Pupo, M.T. (2018) Chemical Signaling Involved in Plant-Microbe Interactions. Chemical Society Reviews, 47, 1652-1704. https://doi.org/10.1039/C7CS00343A

[93] Bhattacaryya, P. and Jha, D. (2012) Plant Growth-Promoting Rhizobacteria (PGPR): Emergence in Agriculture. World Journal of Microbiology and Biotechnology, 28, 1327-1350. https://doi.org/10.1007/s11274-011-0979-9

[94] Etesami, H. and Maheshwari, D.K. (2018) Use of Plant Growth Promoting Rhizobacteria (PGPRs) with Multiple Plant Growth Promoting Traits in Stress Agriculture: Action Mechanisms and Future Prospects. Ecotoxicology and Environmental Safety, 156, 225-246. https://doi.org/10.1016/j.ecoenv.2018.03.013

[95] El-Mohamedy, R.S.R. and Mohamed, S.K. (2018) Effect of Moringa oleifera Seed Oil, Root and Leave Extracts on Growth of Major Pathogenic Fungi of Tomato, Green Bean and Potato in Vitro. International Journal of Agricultural Technology, $14,505-520$.

[96] Tummaramatti, S.H. and Hegde, L. (2016) Effect of Bio-Fertilizers on Growth, Yield and Quality of Buckwheat (Fagopyrum esculentum Moench). Environment \& Ecology, 34, 1258-1261.

[97] Asl, K.K. and Hatami, M. (2019) Application of Zeolite and Bacterial Fertilizers Modulates Physiological Performance and Essential Oil Production in Dragonhead under Different Irrigation Regimes. Acta Physiologiae Plantarum, 41, Article No. 17. https://doi.org/10.1007/s11738-018-2801-x 
[98] Adekiya, A.O., Agbede, T.M., Aboyeji, C.M., Dunsin, O. and Ugbe, J.O. (2019) Green Manures and NPK Fertilizer Effects on Soil Properties, Growth, Yield, Mineral and Vitamin C Composition of Okra (Abelmoschus esculentus (L.) Moench). Journal of the Saudi Society of Agricultural Sciences, 18, 218-223. https://doi.org/10.1016/j.jssas.2017.05.005

[99] Yadav, K.K. and Biofertilizers, S.S. (2019) Impact on Soil Fertility and Crop Productivity under Sustainable Agriculture. Environment \& Ecology, 37, 89-93.

[100] Halpern, M., Bar-Tal, A., Ofek, M., Minz, D., Muller, T. and Yermiyahu, U. (2015) The Use of Biostimulants for Enhancing Nutrient Uptake. In: Advances in Agronomy, Vol. 130, Elsevier, Amsterdam, 141-174. https://doi.org/10.1016/bs.agron.2014.10.001

[101] Canellas, L.P., Olivares, F.L., Aguiar, N.O., Jones, D.L., Nebbioso, A., Mazzei, P. and Piccolo, A. (2015) Humic and Fulvic Acids as Biostimulants in Horticulture. Scientia Horticulturae, 196, 15-27. https://doi.org/10.1016/j.scienta.2015.09.013

[102] Olivares, F.L. (2017) Plant Growth Promoting Bacteria and Humic Substances: Crop Promotion and Mechanisms of Action. Chemical and Biological Technologies in Agriculture, 4, Article No. 30. https://doi.org/10.1186/s40538-017-0112-X

[103] Ahmed, Y.M., Shalaby, E.A. and Shanan, N.T. (2013) The Use of Organic and Inorganic Cultures in Improving Vegetative Growth, Yield Characters and Antioxidant Activity of Roselle Plants (Hibiscus sabdariffa L.). African Journal of Biotechnology, 10, 1988-1996.

[104] Ghasemi, K. (2015) Antioxidant Properties of Garlic as Affected by Selenium and Humic Acid Treatments. New Zealand Journal of Crop and Horticultural Science, 43, 173-181. https://doi.org/10.1080/01140671.2014.991743

[105] Hendawy, S.F., Hussein, M.S., El-Gohary, A.E. and Ibrahim, M.E. (2015) Effect of Foliar Organic Fertilization on the Growth, Yield and Oil Content of Mentha piperita var. citrata. Asian Journal of Agricultural Research, 9, 237-248. https://doi.org/10.3923/ajar.2015.237.248

[106] Shahabivand, S., Padash, A., Aghaee, A., Nasiri, Y. and Rezaei, P.F. (2018) Plant Biostimulants (Funneliformis mosseae and Humic Substances) Rather than Chemical Fertilizer Improved Biochemical Responses in Peppermint. Iranian Journal of Plant Physiology, 8, 2333-2344.

[107] Wu, C.H., Dewir, Y.H., Hahn, E.J. and Paek, K.Y. (2006) Optimization of Culturing Conditions for the Production of Biomass and Phenolics from Adventitious Roots of Echinacea angustifolia. Journal of Plant Biology, 49, 193-199. https://doi.org/10.1007/BF03030532

[108] Masong, P. (2005) Cleaner Production of Essential Oils by Steam Distillation. Journal of Cleaner Production, 13, 833-839. https://doi.org/10.1016/j.jclepro.2004.02.039

[109] Adams, R.P. (1995) Identification of Essential Oil Components by Chromatography/Mass Spectroscopy. Allured Publishing Corporation, Carol Stream, IL.

[110] Moreira, H., Pereira, S.L.A., Vega, A., Castro, P.M.L. and Marques, A.P.G.C. (2020) Synergistic Effects of Arbuscular Mycorrhizal Fungi and Plant Growth-Promoting Bacteria Benefit Maize Growth under Increasing Soil Salinity. Journal of Environmental Management, 257, Article ID: 109982. https://doi.org/10.1016/j.jenvman.2019.109982

[111] El-Kinany, R.G., Salama, Y.E., Rozan, M.A., Bayomy, H.M. and Nassar, A.M.K. (2020) Impacts of Humic Acid, Indole Butyric Acid (IBA) and Arbuscular Mycorr- 
hizal Fungi (Glomus mosseae) as Growth Promoters on Yield and Phytochemical Characteristics of Hibiscus Sabdariffa (Roselle). Alexandria Science Exchange Journal, 41, 29-41. https://doi.org/10.21608/asejaiqjsae.2020.73036

[112] Sharif, M., Khattak, R.A. and Sarir, M.S. (2002) Effect of Different Levels of Lignitic Coal Derived Humic Acid on Growth of Maize Plants. Communications in Soil Science and Plant Analysis, 33, 3567-3580. https://doi.org/10.1081/CSS-120015906

[113] Ibrahim, H.A. and El-Fiki, I.A.I. (2019) Study on the Effect of Yeast in Compost Tea Efficiency in Controlling Chocolate Leaf Spot Disease in Broad Bean (Vicia faba). Organic Agriculture, 9, 175-188.

[114] Aly, E.F.A., Khalil, S.R. and Abdel Fattah, M.E. (2017) Effect of Boron, Potassium and Calcium on Growth, Yield and Quality of Two Sugar Beet Varieties under Sandy Soil Conditions. International Journal of Plant Production, 8, 699-704. https://doi.org/10.21608/jpp.2017.40528

[115] Forttunati, E., Mazzaglia, A. and Balestra, G.M. (2019) Sustainable Control Strategies for Plant Protection and Food Packaging Sectors by Natural Substances and Novel Nanotechnological Approaches. Journal of the Science of Food and Agriculture, 99, 986-1000. https://doi.org/10.1002/jsfa.9341

[116] Yedidia, I., Shoresh, M., Kerem, Z., Benhamou, N., Kapulnik, Y. and Chet, I. (2003) Concomitant Induction of Systemic Resistance to Pseudomonas syringae pv. lachrymans in Cucumber by Trichoderma asperellum (T-203) and Accumulation of Phytoalexins. Applied and Environmental Microbiology, 69, 7343-7353. https://doi.org/10.1128/AEM.69.12.7343-7353.2003

[117] Kamel, S.M., Ebtsam, M.M. and Massoud, O.N. (2016) Potentiality of Some Yeast Species as Biocontrol Agents against Fusarium oxysporum f. sp. cucumerinum the Causal Agent of Cucumber Wilt. Egyptian Journal of Biological Pest Control, 26, 185-193.

[118] Said-Al Ahl, H., Gendy, A.G. and Omer, E.A. (2016) Humic Acid and Indole Acetic Acid Affect Yield and Essential Oil of Dill Grown under Two Different Locations in Egypt. International Journal of Pharmacy and Pharmaceutical Sciences, 8, 146-157.

[119] Lopez-Reyes, J.G., Spadaro, D., Gullino, M.L. and Garibaldi, A. (2010) Efficacy of Plant Essential Oils on Postharvest Control of Rot Caused by Fungi on Four Cultivars of Apples in Vivo. Flavour and Fragrance Journal, 25, 171-177. https://doi.org/10.1002/ffj.1989

[120] Servili, A., Feliziani, E. and Romanazzi, G. (2017) Exposure to Volatiles of Essential Oils Alone or under Hypobaric Treatment to Control Postharvest Gray Mold of Table Grapes. Postharvest Biology and Technology, 133, 36-40. https://doi.org/10.1016/j.postharvbio.2017.06.007 